\title{
GLOBAL LARGE SCALE STRUCTURES IN THE F REGION
}

\author{
S. H. Gross \\ Polytechnic Institute of New York
}

Farmingdale, NY 11735

Measurements of neutral densities, temperatures and ion density and temperature by the Atmosphere Explorer $\mathrm{C}$ and $\mathrm{E}$ satellites exhibit fluctuations made up of coherent waves of scale size from hundreds to thousands of kilometers. The fluctuations in the AE-C data are found at times of low magnetic activity to extend from the auroral region in one hemisphere across the equator to the corresponding region in the other hemisphere with little change in apparent wavelength. The fluctuations in the AE-E data similarly exhibit at times of low magnetic activity nearly uniform wave structure encircling the equatorial belt. Typical examples of waves of more than $1000 \mathrm{~km}$ apparent wavelength in both the AE-C and AE-E data will be presented. These observations during quiet times may be explained by a system of quasi-stationary waves excited by the auroral belts that are distributed about the earth possibly with tidal or sub-tidal periods and scale lengths determined by the structure of the belts. According to this interpretation, TID's are manifestations of transient wave systems moving past a ground observatory that result from changes in magnetic activity with periods and scale sizes associated with the regions of enhanced auroral activity. Some evidence suggesting tidal-like stationary waves from the Millstone Hill incoherent scatter radar is presented.

The first eight figures that follow are for AE-C. The next four are for AE-E and the last is for Millstone Hill incoherent scatter radar measurements. It will be seen for the fluctuations in the data measured by AE-C that there is little variation in the scale size of the structures while the satellite is moving from one hemisphere to the other. Since the orbit is inclined $68^{\circ}$, there are portions of the orbit when the satellite is at high latitudes moving almost west to east. There is no reason why the behavior of the wave structure for these parts of the orbit should be the same as the parts in which the satellite is moving mostly from north to south or from south to north. Furthermore, as one might expect with fixed wavelength waves, there is no evidence of exponential attenuation with distance as might occur due to loss processes. The mode of propagation, if these are propagating waves, must be such as to incur low losses.

Figure 1 is a plot of AE-C measured data on January 20, 1975. The orbit was nearly circular, inclined $68^{\circ}$ and the satellite was at an altitude of about $250 \mathrm{~km}$. Densities of atomic oxygen, nitrogen, helium and the electron density ( $\mathrm{Ne}$ in the figure) are plotted in the upper graph. The lower graph contains the electron temperature (dotted line) and the ion temperature (heavy line). These are 15 seconds, United Abstract file data. (All the AE examples shown here are from these files, including AE-E). Magnetic activity was quite moderate on this day.

Figure 2 is a plot of fluctuations obtained for the data of Figure 1. The data are normalized, detrended and passed through a high pass filter that passes all waves with wavelengths less than $2600 \mathrm{~km} .2000 \mathrm{~km}$ waves are evident in the figure. Three pairs of fluctuations are shown. The solid 
line in each is for oxygen for comparison, the other in each, shown dotted, is as indicated. The three nearly even peaks in the oxygen fluctuations near the start from just before 29760.0 seconds UT to about 29760.0 seconds are for the part of the orbit where the satellite is at high latitudes moving west to east. From 29760.0 seconds to about mid-way between 30585.0 and 31410.0 seconds there are five nearly even spaces between peaks in the oxygen fluctuations. Similar behavior is evident in the nitrogen fluctuations and the phase shifted ion temperature fluctuations. During this time the satellite was moving southward from just below $60^{\circ} \mathrm{N}$ to $-30^{\circ} \mathrm{S}$. Beyond, there is evidence of nearly equal spacing, but the magnitude is diminished. At 31410.0 seconds the satellite is again traversing mostly from west to east in the southern hemisphere. Exponential damping is not evident.

Figure 3 contains nitrogen fluctuations for AE-C measurements on January 19, 1975, a day of moderate magnetic activity in which $k_{p}$, did not exceed $3_{o}$. The same filter as for Figure 2 is employed. The satellite is at high latitudes moving mostly from west to east until just before 62481.0 seconds UT when it starts moving southward. There are roughly evenly spaced waves of $2000 \mathrm{~km}$ scale size until a time midway between 64173.0 and 65019.0 seconds UT. At this point the satellite has reached southern high latitudes and is moving mostly from west to east before reversing its path and returning to the nothern hemisphere.

Figure 4 is for the same orbit as Figure 3, but the data were passed through a high pass filter that passes only waves with wavelengths less than $1300 \mathrm{~km}$. Waves of the order of $900 \mathrm{~km}$ are evident. The north to south region is again from about 62481.0 seconds to midway between 64173.0 and 65019 seconds. More uniformity appears in the region from this midpoint (roughly $-60^{\circ} \mathrm{S}$ ) to earlier times, a point somewhat to the left of 63327.0 seconds (at about $30^{\circ} \mathrm{N}$ ).

Figure 5 is for another day of AE-C data, February 17, 1975. The densities of nitrogen (top curve), helium (second highest), argon (heavy line) and electron density (thin curve varying about the argon curve after 36232.5 seconds) are plotted. Two auroral zone heating signatures are evident, one very distinct on the left at high northern latitudes, centered somewhat to the left of 34856.3 seconds, and a second, less distinct, at high southern latitudes centered to the left of 37608.8 seconds. The heating in both regions may act as wave sources. The $k_{p}$ for this day did not exceed $3_{+}$.

Figure 6 is a plot for the fluctuations of nitrogen (solid line) and argon (dotted line) for the data in Figure 5. It was obtained by passing the data through a bandpass filter allowing only waves in the range 1100 to $2600 \mathrm{~km}$ to pass. Strong waves to the left of 34971.0 seconds are for the northern auroral region. Similar strong waves are observed in the southern auroral region to the left of 37509.0 seconds. The equator is crossed at about 35817.0 seconds. The pattern looks like that of two interfering wave systems. High latitude west to east traversal in the northern hemisphere is for the time interval 34125.0 to 34971.0 seconds, and somewhat to the right of 36663.0 to somewhat past 37509.8 seconds for the southern hemisphere.

Figure 7 contains the cross correlation function for the fluctuations of Figure 6. The large negative peak of -.8 shows that argon and nitrogen are well correlated and that a coherent wave system exists.

Figure 8 contains fluctuations for the data of Figure 5 but for a bandpass filter passing waves in the range 800 to $1300 \mathrm{~km}$. These are roughly $1000 \mathrm{~km}$ waves of nitrogen fluctuations. The southern region at about 36663.0 seconds is stronger by a factor of about 2 . The fluctuations resemble that of two interfering waves, perhaps from two sources. Fairly evenly spaced waves are evident between 34971.0 and 36663.0 seconds.

Figure 9 is a plot similar to that of Figure 1, but is for AE-E measurements on August 1, 1977. AE-E was in a nearly circular orbit inclined $20^{\circ}$ at an altitude of about $260-270 \mathrm{~km}$. The upper graph contains densities of oxygen (upper curve), nitrogen (second highest), helium (third curve) 
and the electron density (lowest). The lower graph contains ion temperature (dotted line) and neutral gas temperature (heavy line). Magnetic activity was moderate; $k_{p}$ did not exceed 3 . The satellite was mainly moving from west to east in an equatorial belt between $\pm 20^{\circ}$.

Figure 10 contains plots of four pairs of fluctuations for the data of Figure 9 as obtained from a high-pass filter passing only waves with wavelengths less than $2600 \mathrm{~km}$. The scale size of the waves is about $1800 \mathrm{~km}$. The solid line in all four graphs is for oxygen, the others (dotted) as indicated. The time shown is for about $3 / 4$ of an entire orbit, limited due to the absence of data prior to and after the orbit. Nearly uniform waves are evident girdling this much of the equatorial belt.

Figure 11 is for AE-E for November 23, 1977. The satellite was in the same orbit as in Figure 9. Neutral and ion winds are shown as well. Magnetic activity was low for this day, $k_{p}$ did not exceed $1_{o}$.

Figure 12 is a plot of fluctuations for the data of Figure 11. Here the data were passed through a bandpass filter allowing only waves in the range 2600 to $5200 \mathrm{~km}$ to pass. The waves in the figure are roughly of $2800 \mathrm{~km}$ in scale size. The upper graph is for neutral temperature and nitrogen density fluctuations. Its cross correlation function is to its right and shows a negative peak of about -.85. Fluctuations of horizontal (roughly north-south) neutral winds and nitrogen density are shown in the lower graph. Its cross correlation function is to its right and contains a negative peak of about -.68 . The time period covers about one half of an orbit, the limitation being the extent of the measurements.

Figure 13 is for the data of Figure 11, but the fluctuations are obtained from a high-pass filter that passes all wavelengths less than $1300 \mathrm{~km}$. These waves are about $1000 \mathrm{~km}$ in scale size and the plot covers about half the equatorial belt. The uniformity of these waves is quite remarkable. The solid line is for nitrogen and the dotted for the neutral temperature.

Figure 14 is a plot of data obtained from a series of Millstone Hill incoherent scatter radar measurements while in an elevation scan mode from due south to due north. The scan is completed in about 20 minutes, but the scan to scan period is 45 minutes. Shown here are the so-called raw fluctuations for individual scans plotted with separations so that they can be seen relative to each other. Not all scans are shown. The plots are to a scale which is not shown. The abscissa is the geomagnetic latitude. The Millstone Hill station is at $54^{\circ}$. The curves shown are constant altitude electron densities at $350 \mathrm{~km}$. The day and beginning time of each scan is shown on the left of each curve. The nature of the variation appears to change from flat to concave to flat to convex, etc., with a period that may relate to the diurnal or semi-diurnal period and for a half wavelength of about $1000 \mathrm{~km}$, much like the behavior of a stationary wave. There is an unfortunate four hours 45 minutes gap between th 03:11 and 07:55 curves due to a radar malfunction. The change in shape for these two times is quite interesting. Whether these variations can be interpreted as evidence of tidal like, stationary variations is highly speculative at this time. Measurements on other days are also somewhat like these curves. 

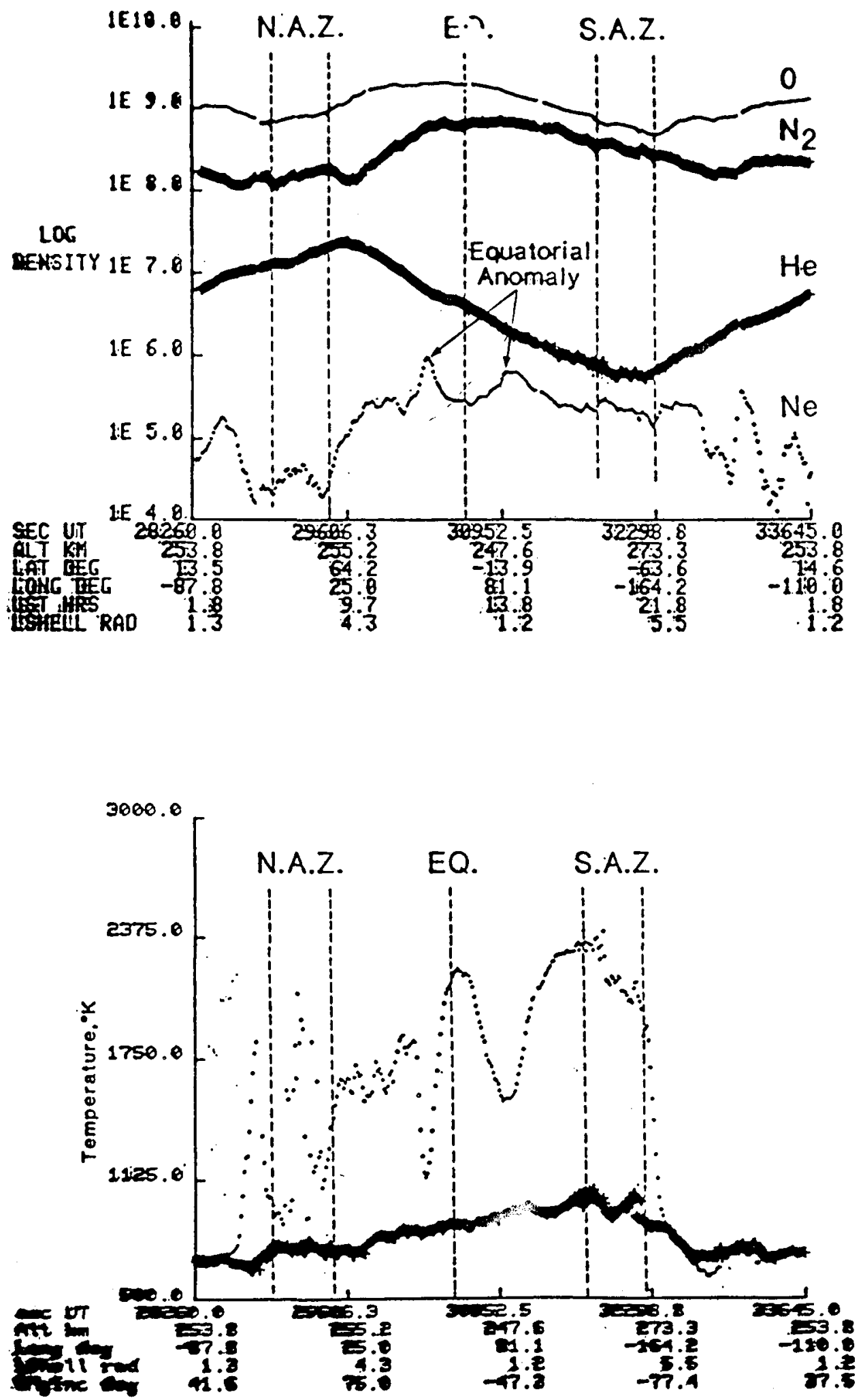

Figure 1. 

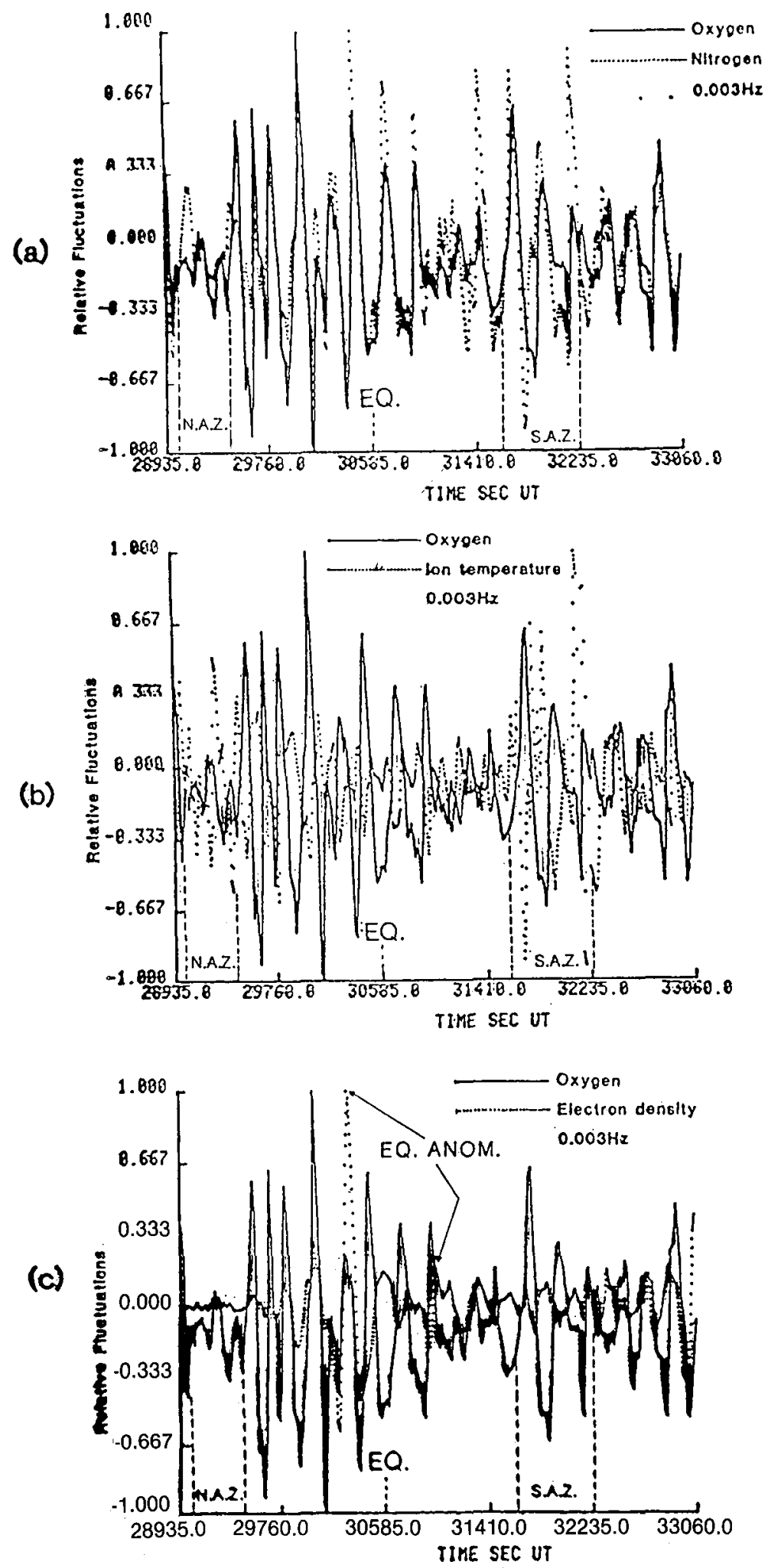

Figure 2. 


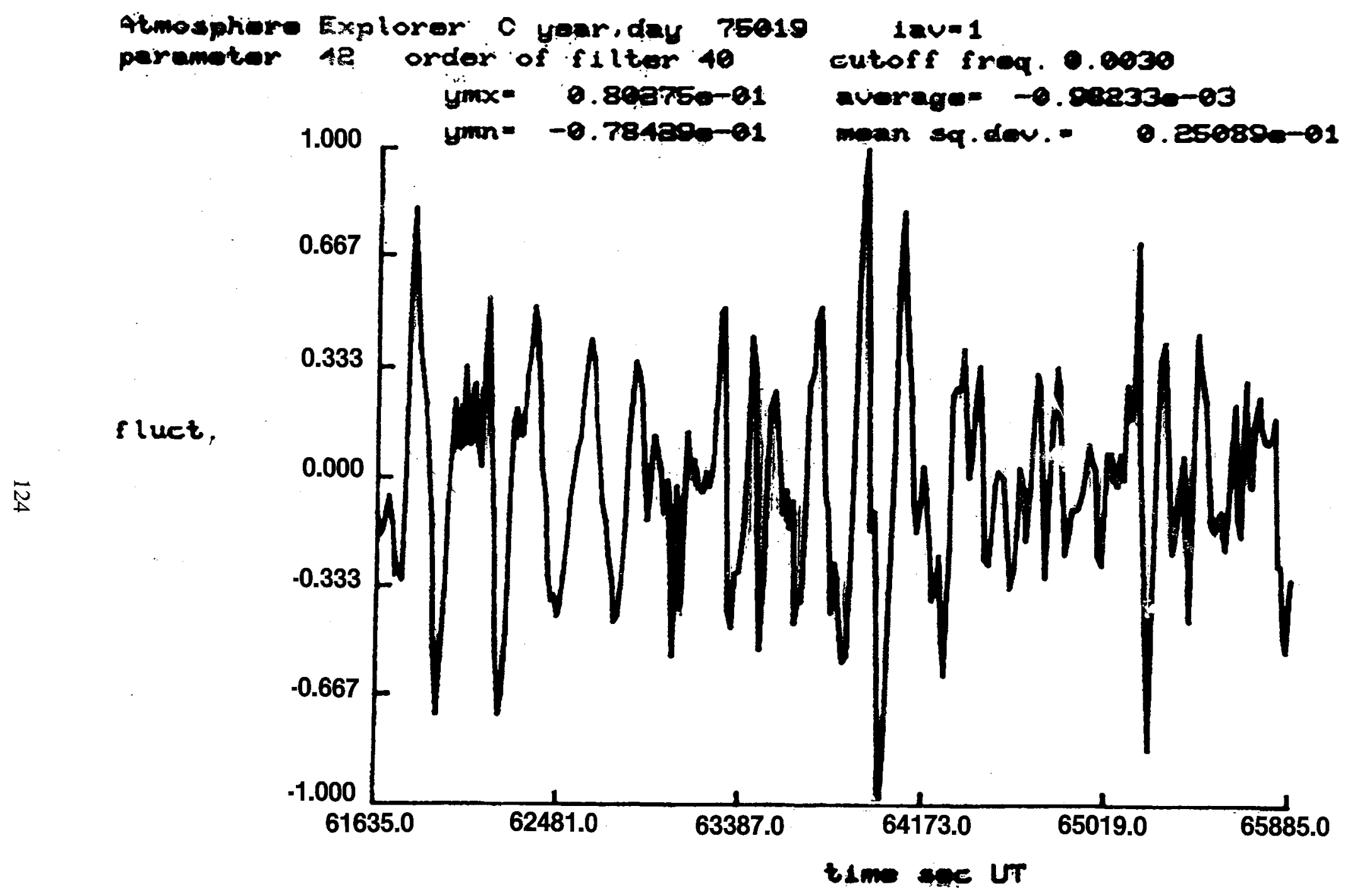

Figure 3. 


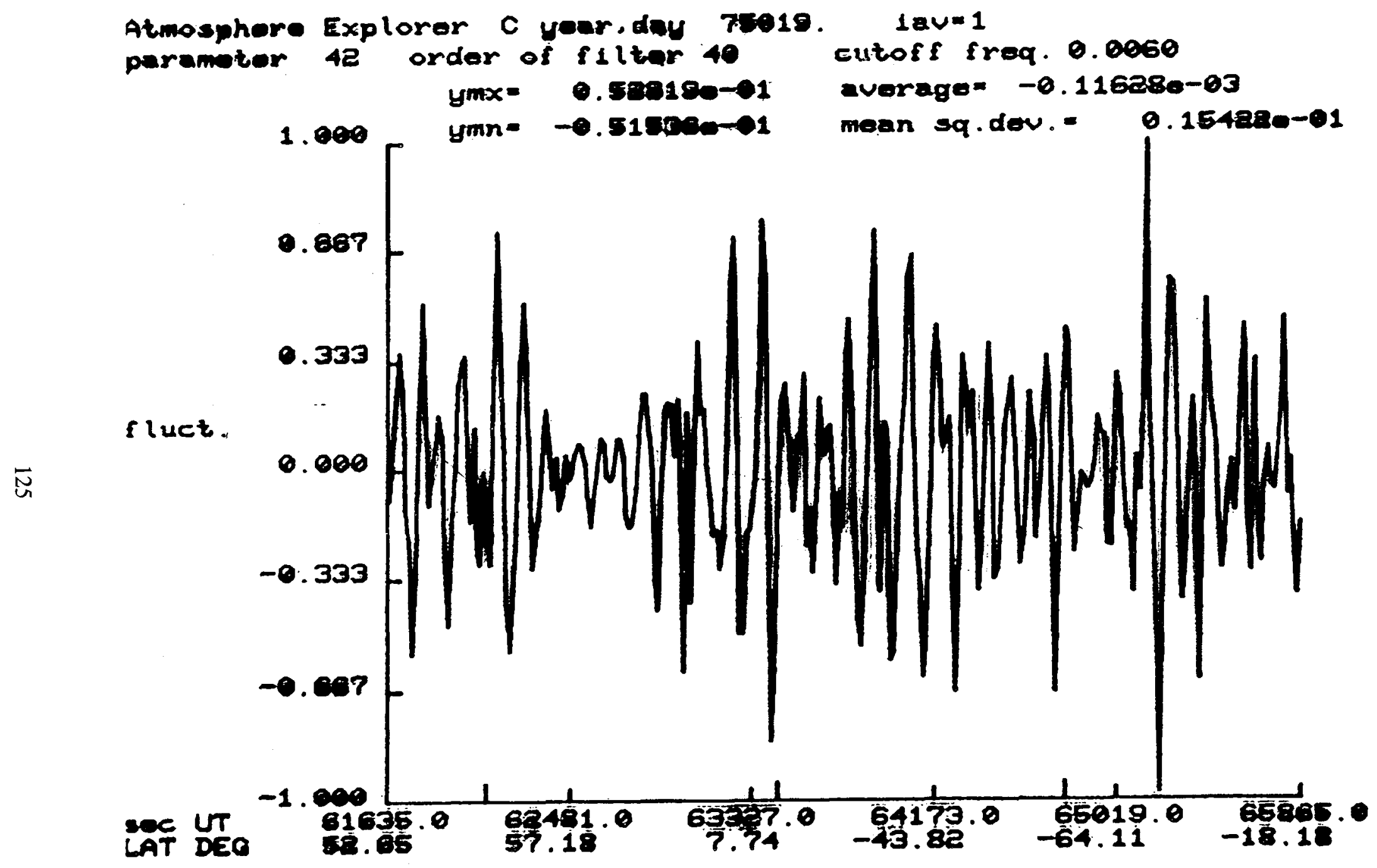

Figure 4. 


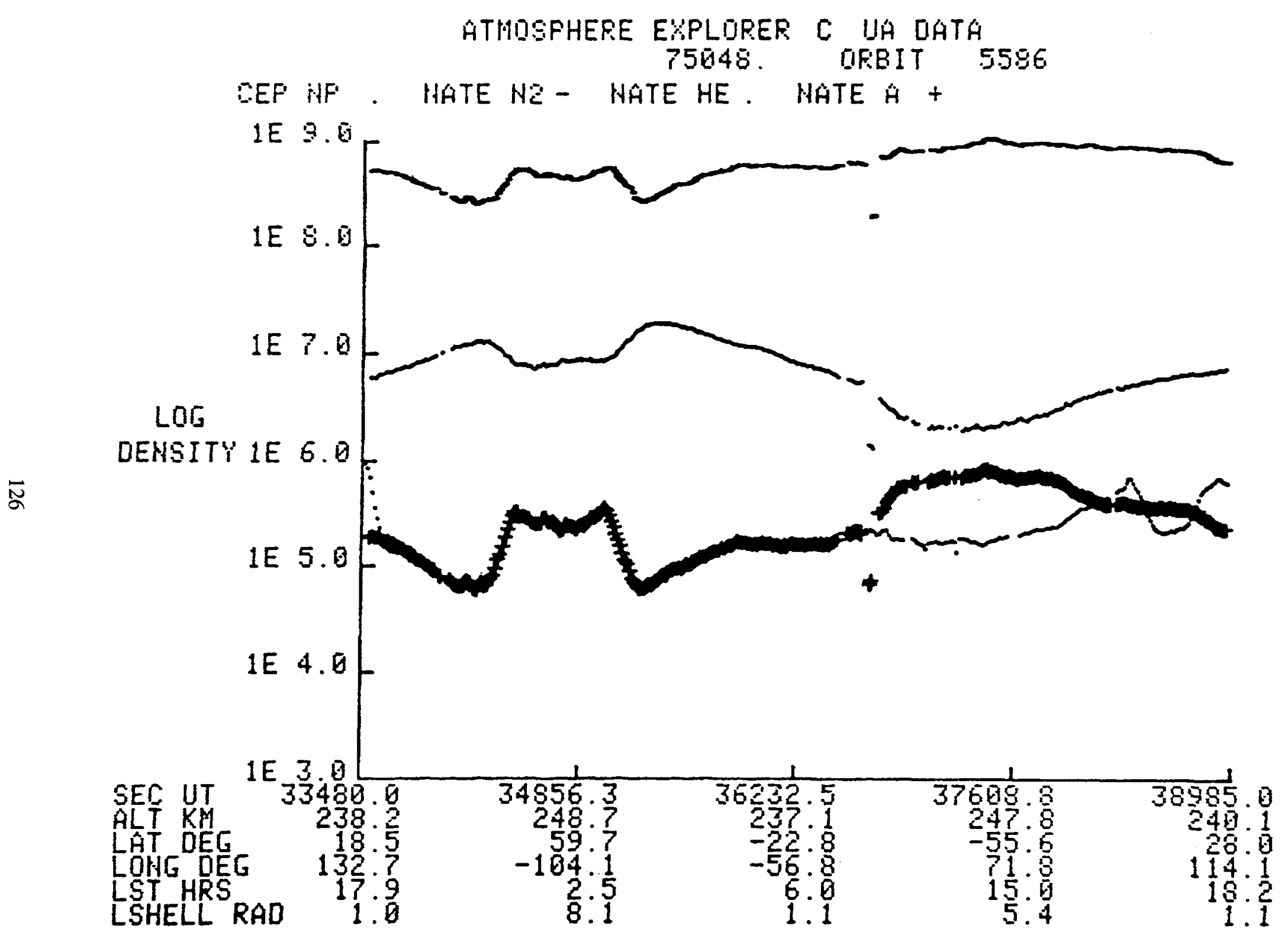

Figure 5. 


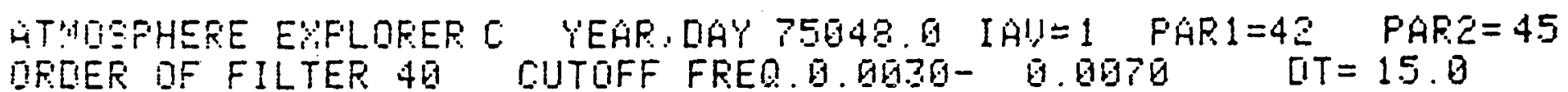

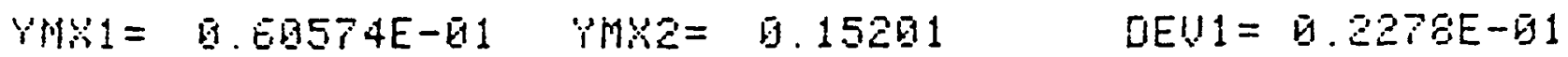

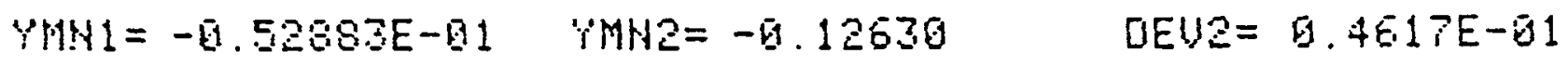

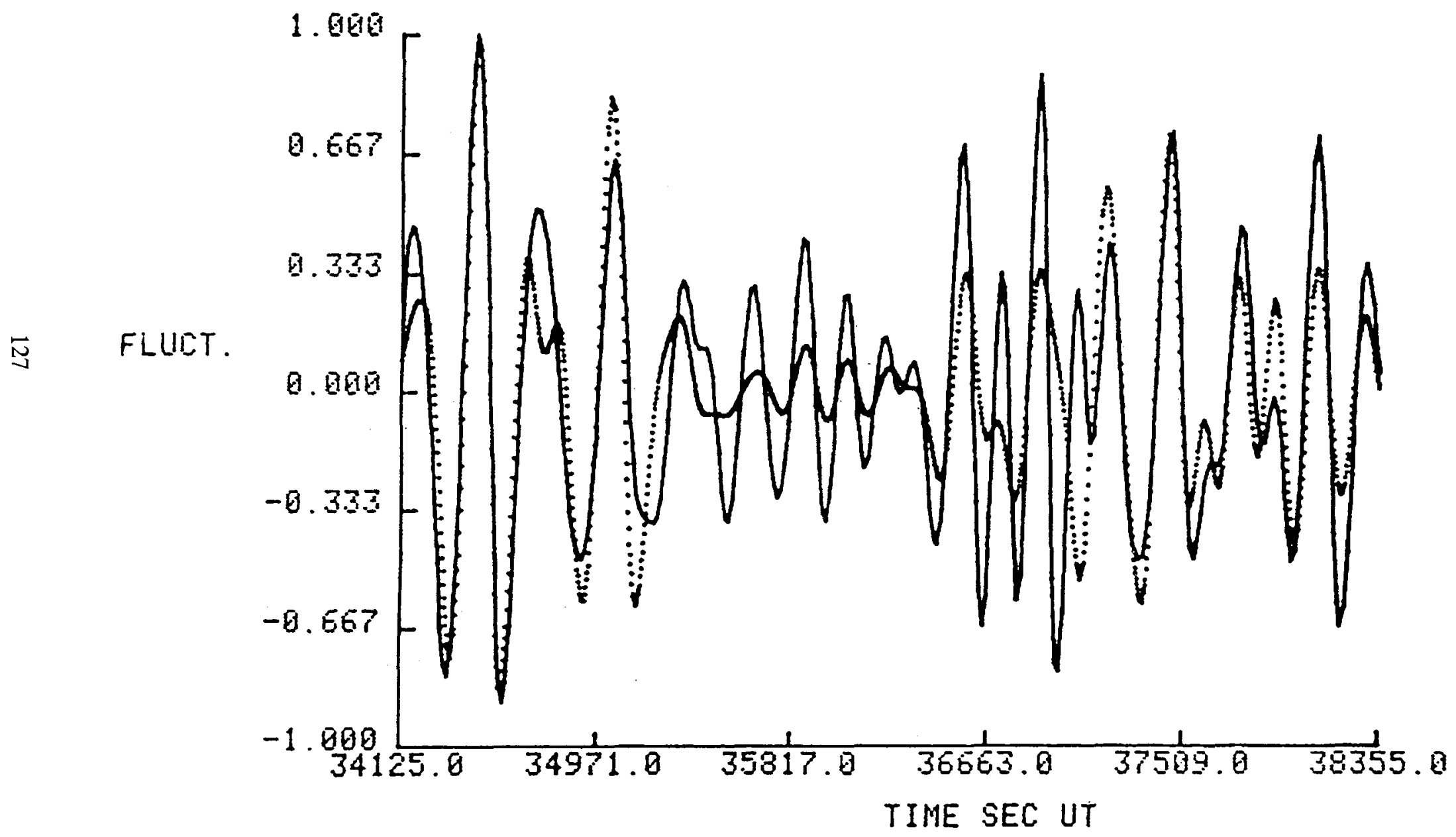

Figure 6. 


\begin{tabular}{|c|c|c|c|}
\hline $\begin{array}{l}\text { ATMOSPHERE ESFLORER } \\
\text { PAR } 142 \text { PAR2 } 45\end{array}$ & $\begin{array}{c}\text { E YEAF: DAY } 75048.0 \\
\text { AU1 }=0.2738 E-0.3\end{array}$ & $\begin{array}{l}34125.0-35355.0 \\
34125.0-38555.0\end{array}$ & \\
\hline OROER OF FILTER $4 \bar{\theta}$ & $\dot{A} \cup Z=0.3652 \mathrm{E}-\overline{6} 3$ & DEU1 = & 0.2278 \\
\hline C.UTOFF FREQ. B. B030- & 0.0070 & DEU2= & D. $4 E 17 E-01$ \\
\hline
\end{tabular}

$I A U=1 \quad \square T=15.060$

$\bar{\infty}$

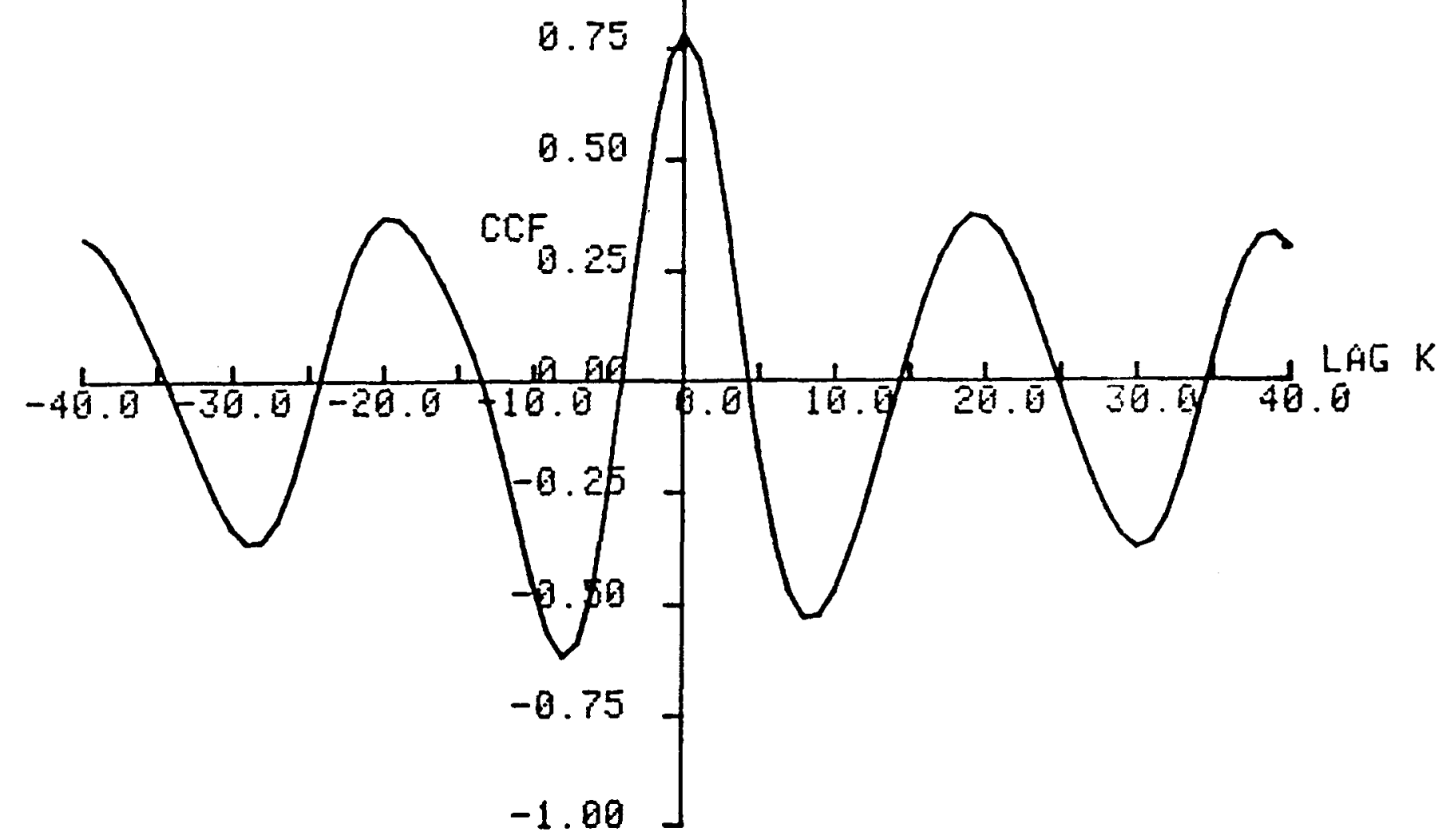

Figure 7. 


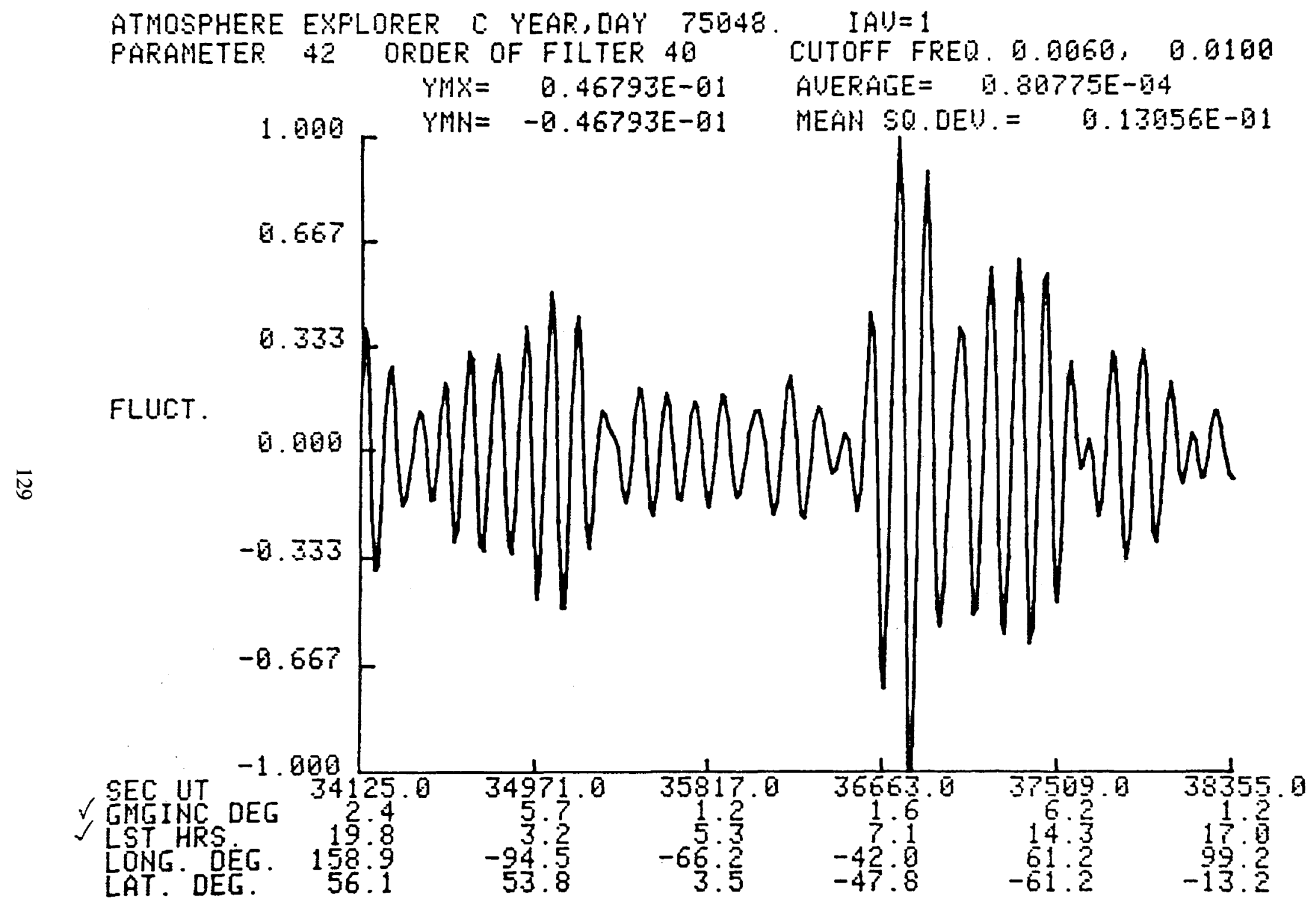

Figure 8. 


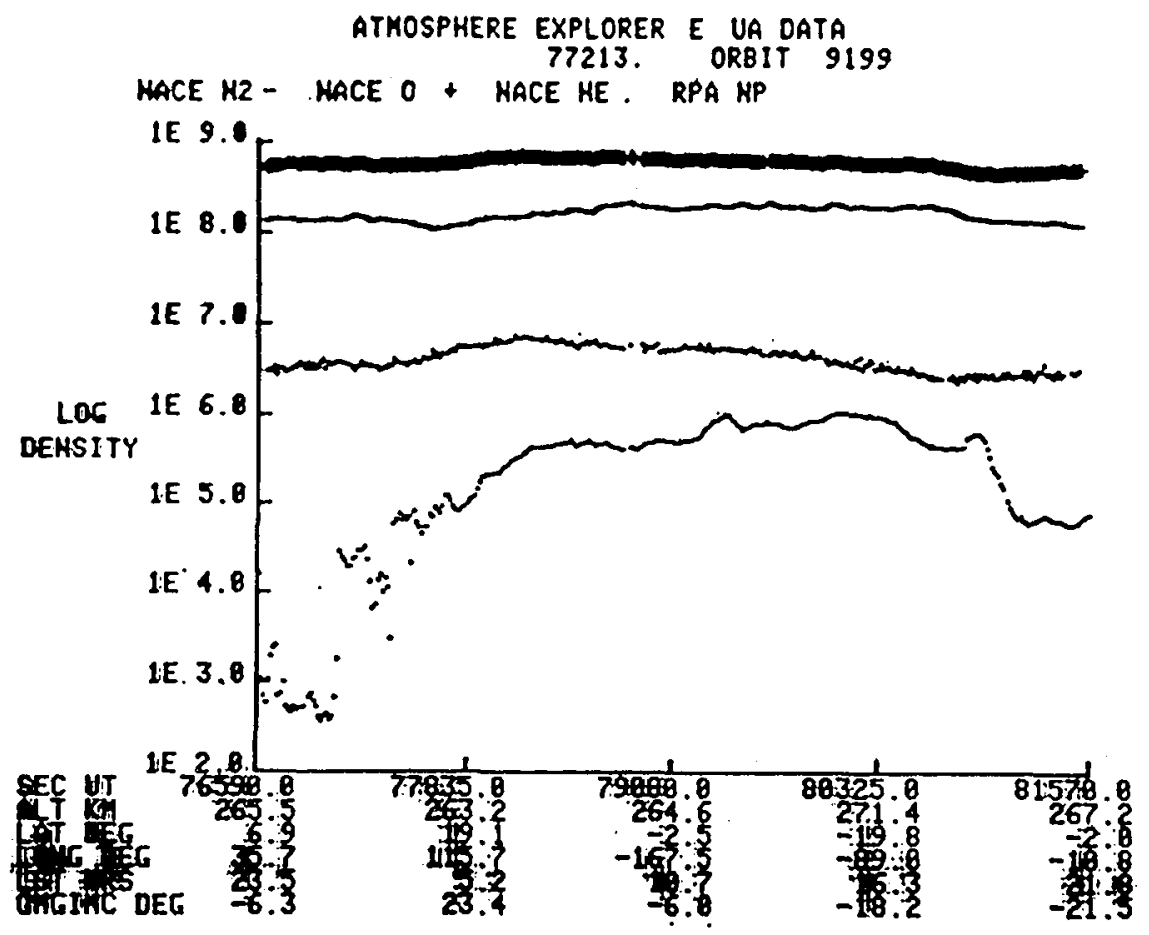

\& ATMOSPHERE EXPLORER E UA BATA

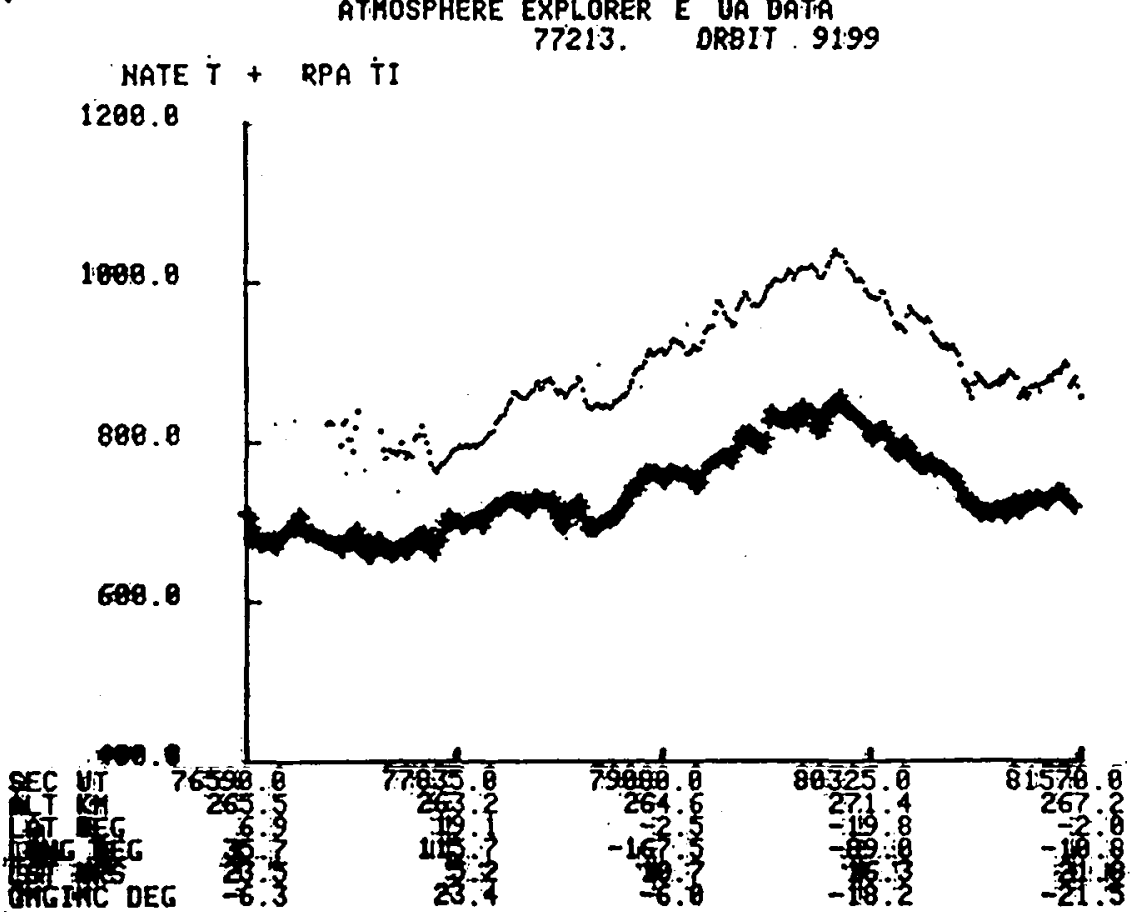

Figure 9. 


\section{AE-E $\quad 77213$ Orbit 9199}
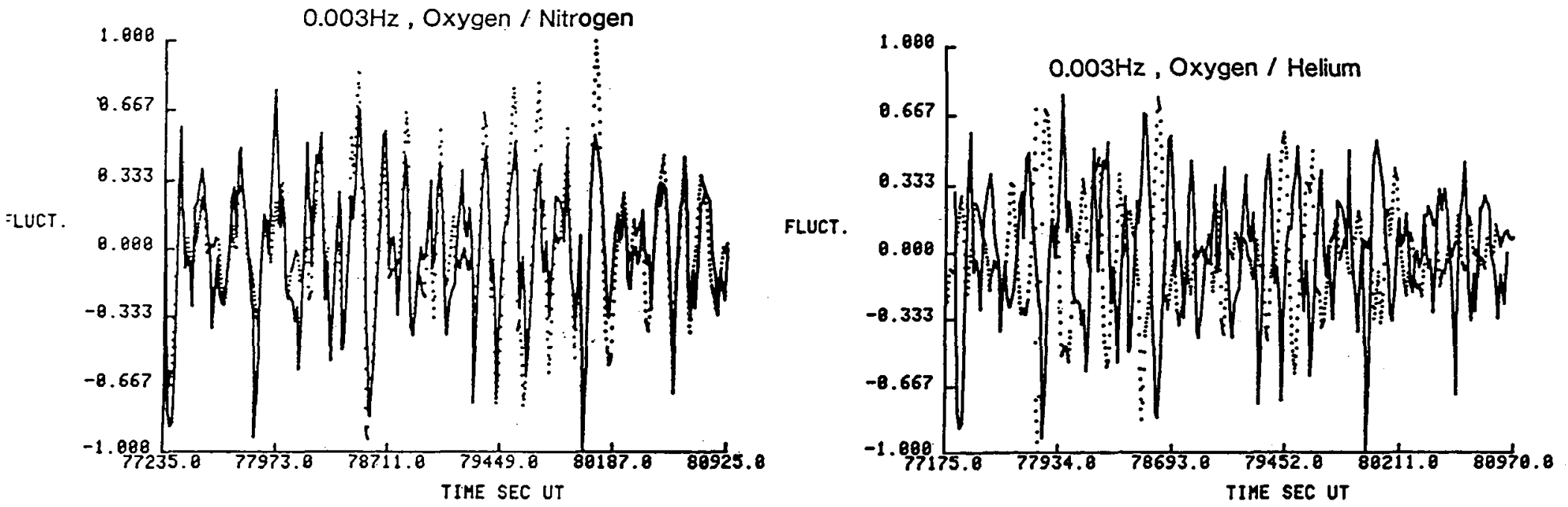

$\omega$
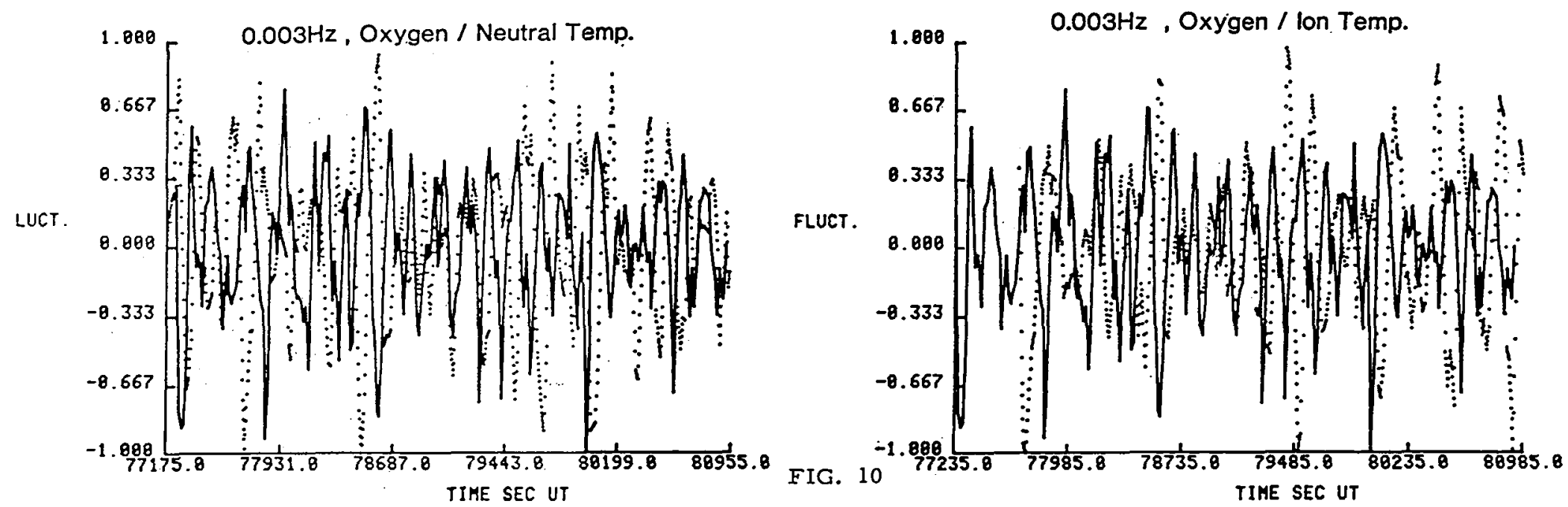

Figure 10. 


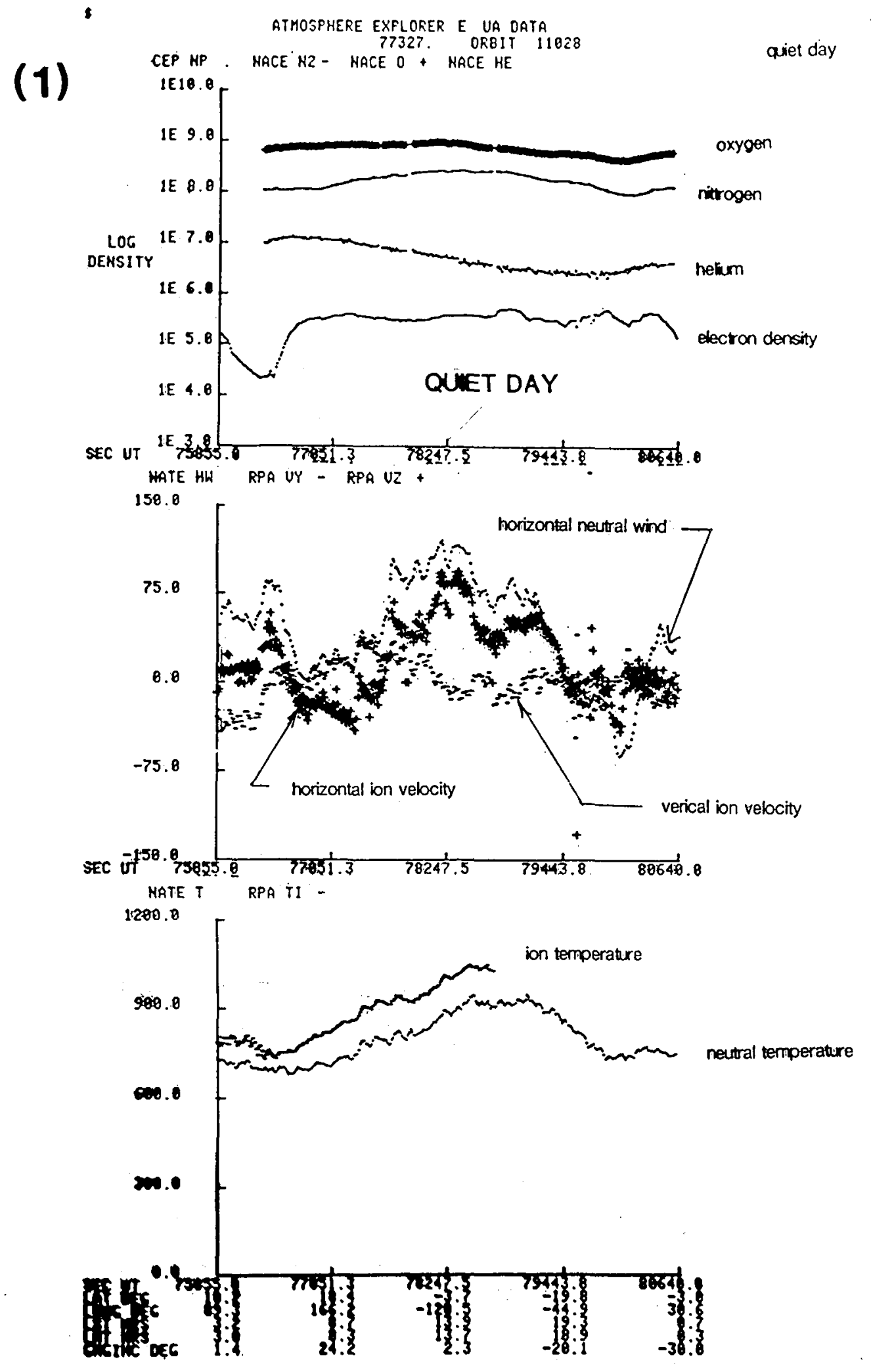

Figure 11. 


\section{NEUTRAL-NEUTRAL CORRELATION}

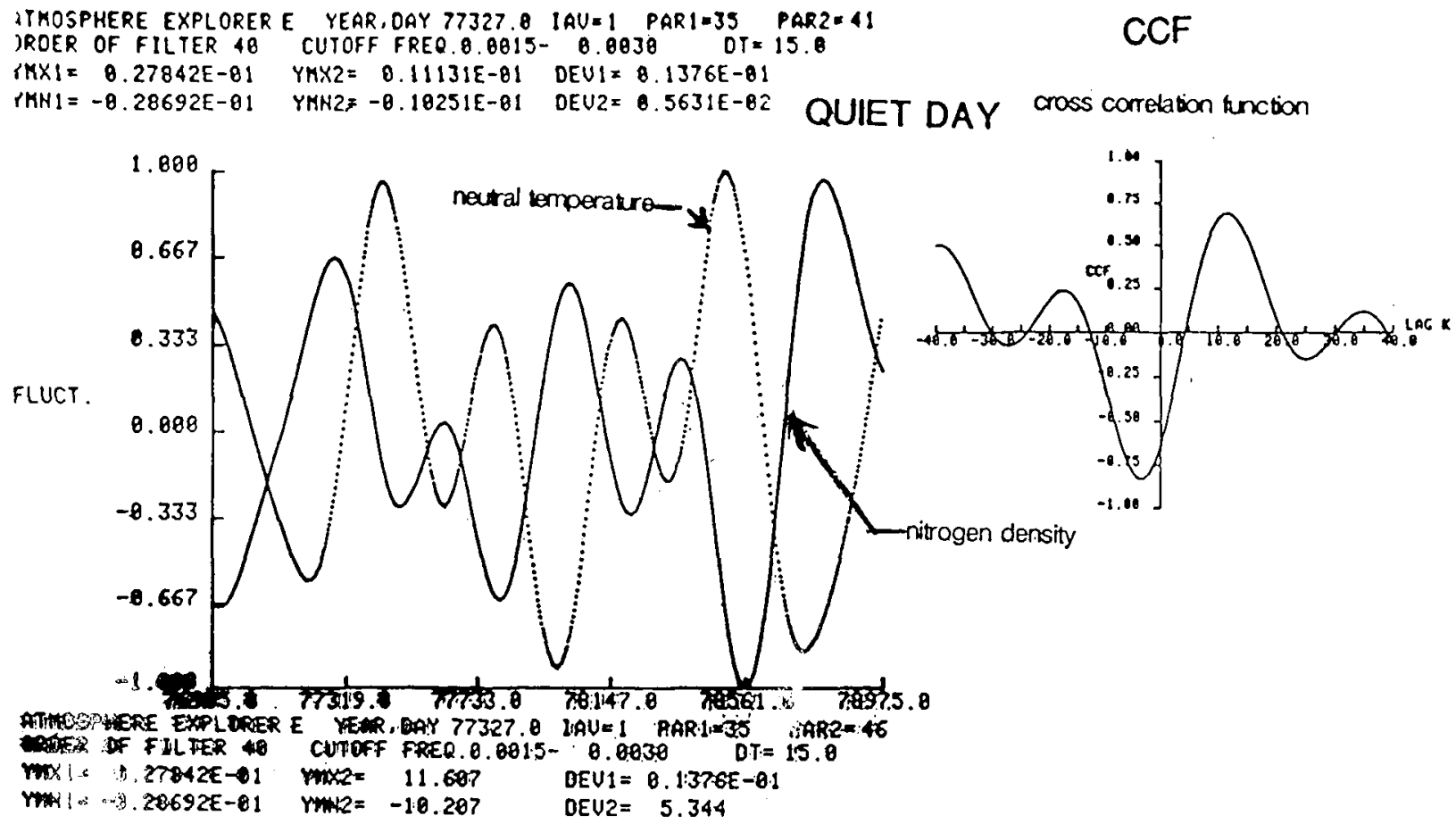

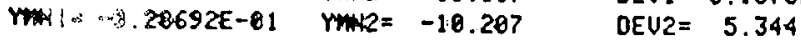

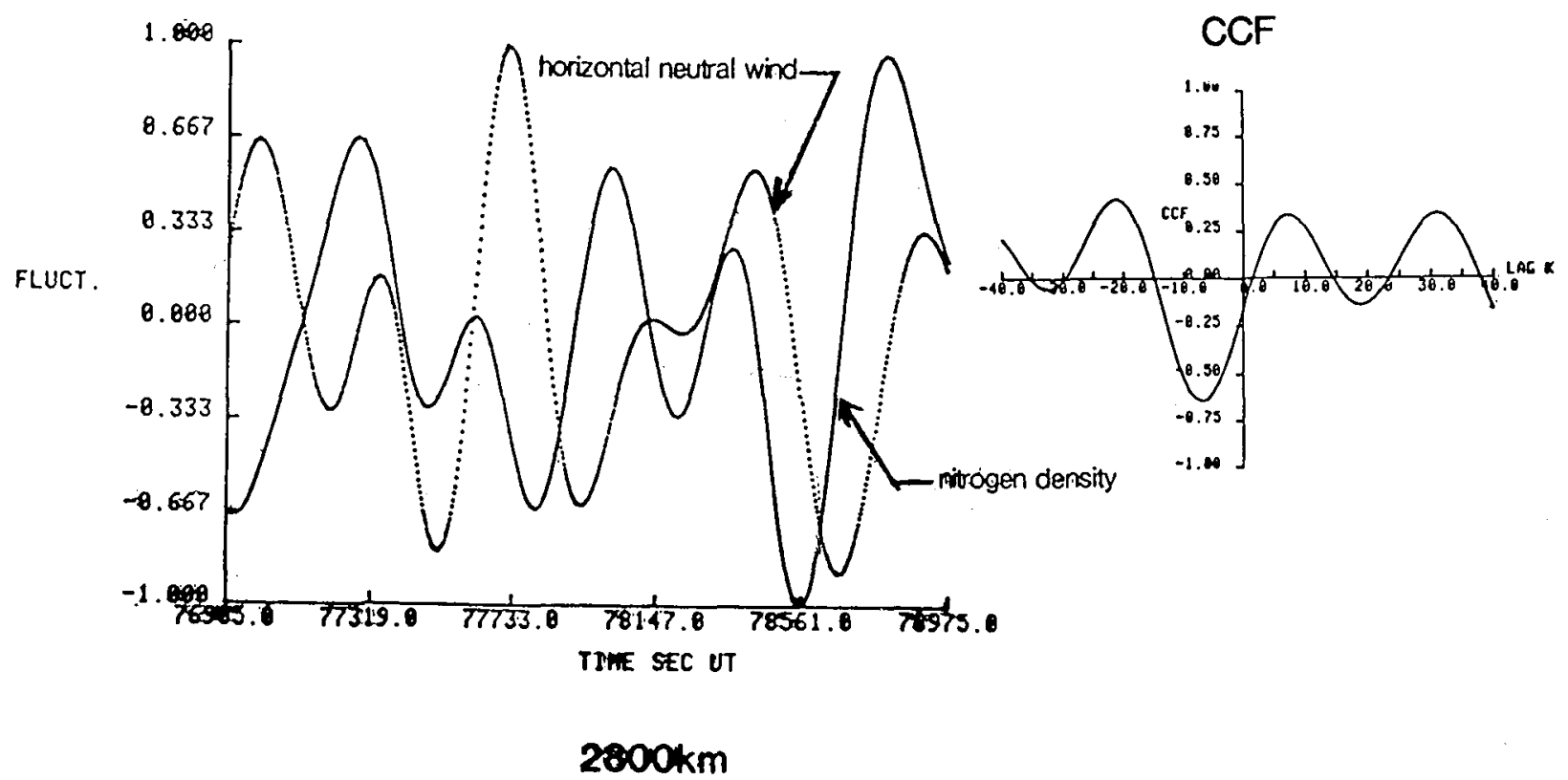

Figure 12. 
ATMOSPHERE EXPLORER E YEAR, DAY 77327.0 IAU $=1$ PAR $1=35$ PAR2 $=41$ OROER OF FILTER 40 CUTOFF FREQ.0.0060

$Y M X 1=0.24831 E-01$ YMY2 $=0.13097 E-01 \quad$ DEU1 $=0.1030 E-01$

YMN $1=-0.25775 E-01 \quad Y M N 2=-0.12704 E-01 \quad$ DEU2 $=0.5626 E-02$

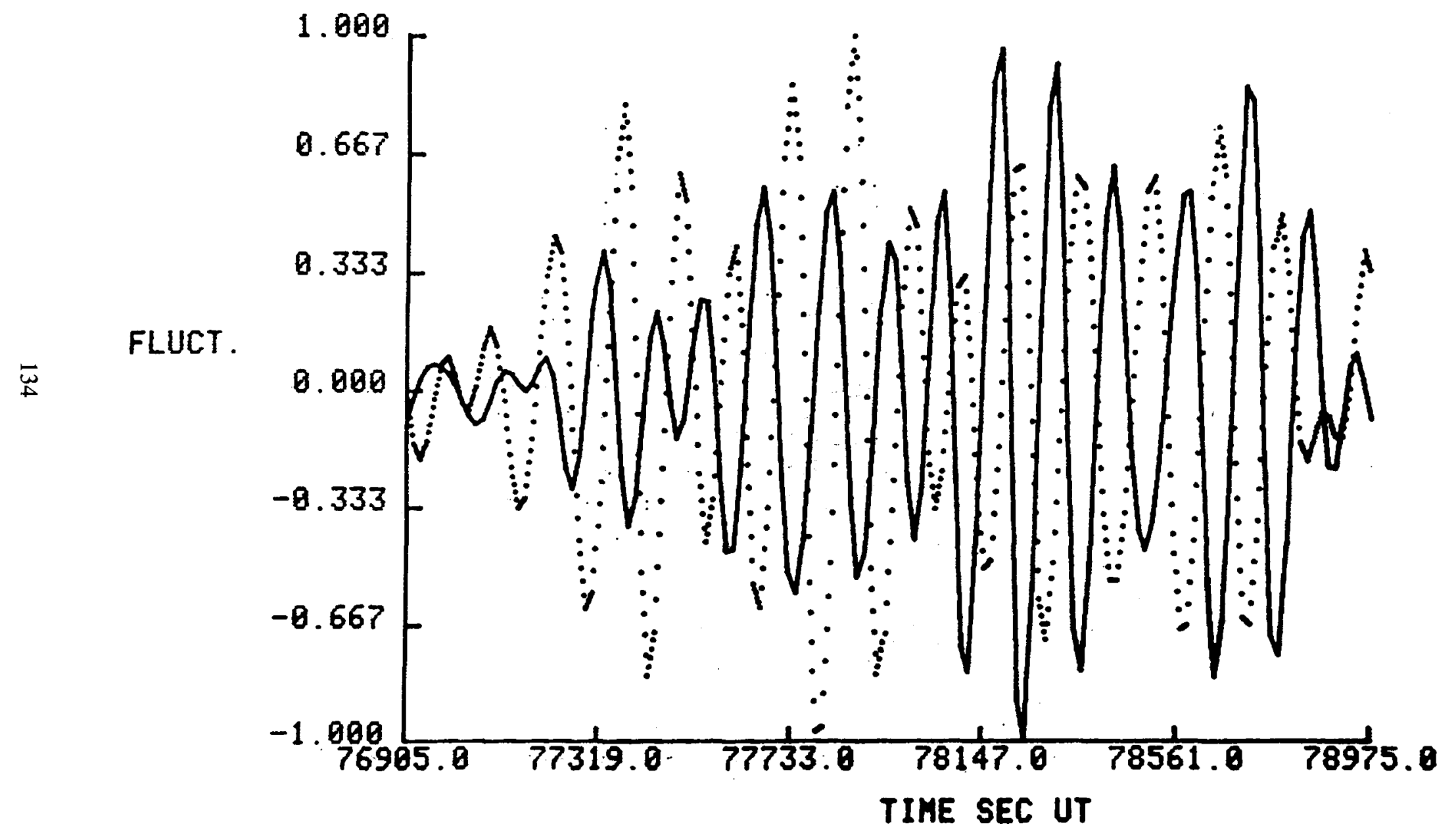

Figure 13. 


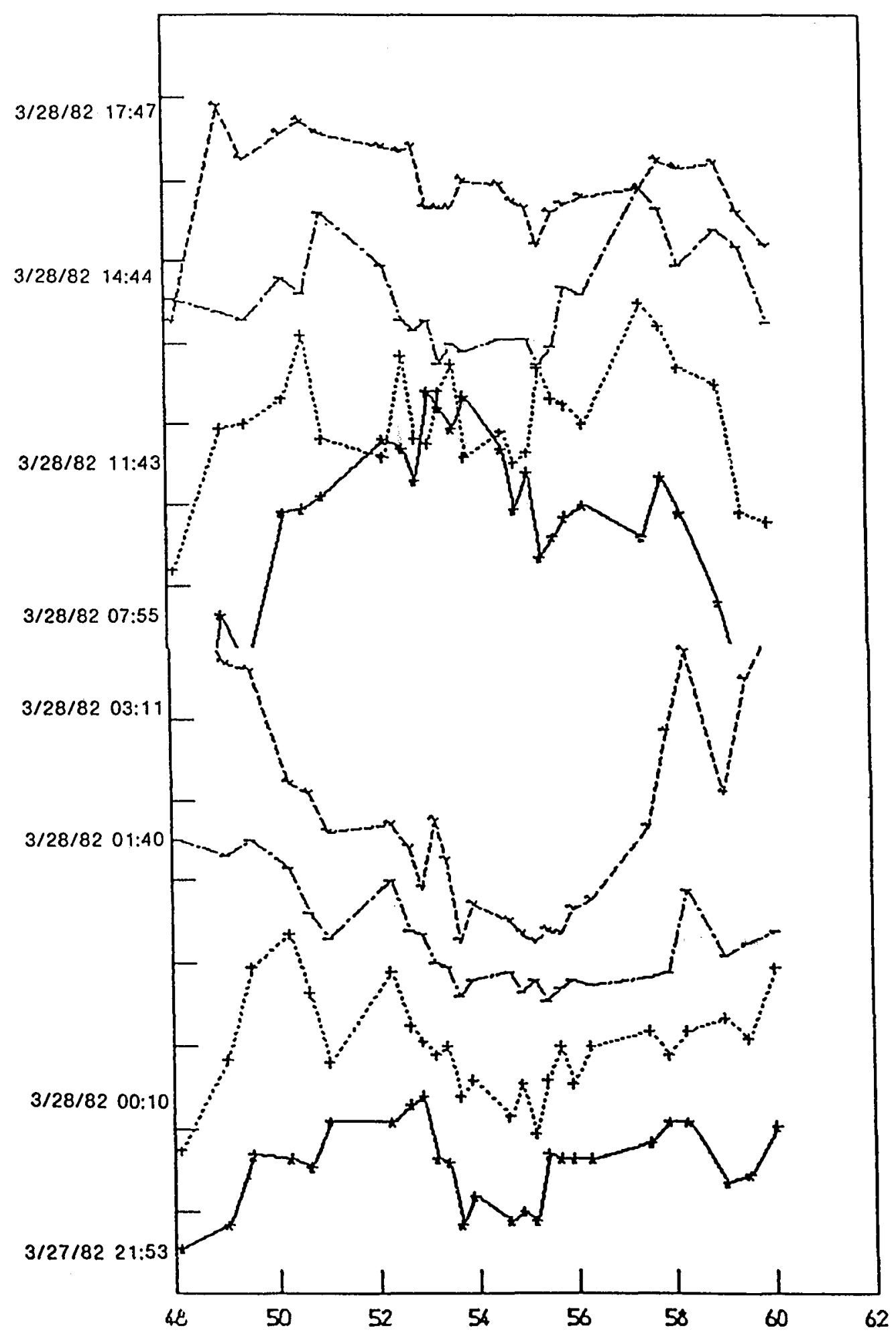

Figure 14. 